\title{
Proceedings of the Thirteenth European Powder Diffraction Conference
}

This special issue of Powder Diffraction offers a selection of contributions presented at the European Powder Diffraction Conference (EPDIC) held in Grenoble, France on October 28-31, 2012 (EPDIC-13).

EPDIC is the only European conference completely dedicated to all aspects of the analysis of polycrystalline materials by diffraction methods. Started in Munich, Germany in 1991, EPDICs have rapidly become reference points for all researchers in the field and are now regarded to be ideal venues for the presentation and diffusion of new developments in powder diffraction instrumentation, analysis, and applications. The next event (EPDIC-14) will take place in Aarhus, Denmark, on June 15-18, 2014 (http://epdic14.au.dk/). Details on the EPDIC organization can be found at the URL: http://epdic.ing.unitn.it/index.html.

As with all EPDIC conferences, EPDIC-13 presented a rich variety of topics, ranging from crystallography and structural studies to materials science and the latest developments in solid state physics and chemistry, with increasing interest in biological, pharmaceutical and biomedical areas. Major events where the plenary talks on Total Scattering Techniques (by D. Keen) the Rietveld Method (by J. Rodriguez-Carvajal), Instrumental Developments of X-FELs (by H. Chapman) Nanoimaging with Synchrotron Radiation X-rays (by V. Favre-Nicolin), Materials Science Applications to Hydrogen Storage (by M.H. Sorby) and Charge Density Analysis (by B. Brummerstedt-Iversen). Special interest was centered on the invited talks presented by the winners of the EPDIC awards for Young Scientists (Kenneth R. Beyerlein, "At the limits of diffraction, from the very small to the very fast"), and for Distinguished Powder Diffractionists (Christian Baerlocher, "The lucky powder diffractionist", a talk on his lifelong research activity).

The remainder of the program was organized in twelve micro-symposia, each providing key-note talks, contributed oral presentations as well as posters, and the Software Fayre, an initiative of past EPDICs now dedicated to the memory of Lachlan Cranswick. There were about 350 registered participants from 37 countries, including 16 countries from outside Europe. EPDIC 13 demonstrated the vitality of the powder diffraction community in Europe and the world, and highlighted the continued interest in diffraction methods and applications.

The guest editors of this special issue of Powder Diffraction

Michela Brunelli, ILL, Grenoble, France

Pierre Bordet, CNRS, Grenoble, France

Paolo Scardi, University of Trento, Trento, Italy 